Incorporating anisotropic electronic structure in crystallographic determination of complex metals: iron and plutonium

K. Moore, D. Laughlin, P. Soderlind, A. Schwartz

November 4, 2005

Philosophical magazine 
This document was prepared as an account of work sponsored by an agency of the United States Government. Neither the United States Government nor the University of California nor any of their employees, makes any warranty, express or implied, or assumes any legal liability or responsibility for the accuracy, completeness, or usefulness of any information, apparatus, product, or process disclosed, or represents that its use would not infringe privately owned rights. Reference herein to any specific commercial product, process, or service by trade name, trademark, manufacturer, or otherwise, does not necessarily constitute or imply its endorsement, recommendation, or favoring by the United States Government or the University of California. The views and opinions of authors expressed herein do not necessarily state or reflect those of the United States Government or the University of California, and shall not be used for advertising or product endorsement purposes. 


\title{
Incorporating anisotropic electronic structure in crystallographic determination of complex metals: iron and plutonium
}

\author{
Kevin T. Moore ${ }^{1 *}$, David E. Laughlin ${ }^{2}$, Per Söderlind ${ }^{3}$, Adam J. Schwartz ${ }^{3}$ \\ ${ }^{1}$ Chemistry and Materials Science, Lawrence Livermore National Laboratory, Livermore, \\ California 94550, USA. \\ ${ }^{2}$ Materials Science and Engineering Department, Carnegie Mellon University, \\ Pittsburgh, PA 15213 USA. \\ ${ }^{3}$ Physics and Advanced Technologies, Lawrence Livermore National Laboratory, \\ Livermore, California 94550, USA.
}

\begin{abstract}
Anisotropic electronic structure is incorporated in crystallographic determination of the structure of ferromagnetic $\alpha-\mathrm{Fe}$ and $\delta$-Pu. This is achieved by using anisotropic aspects of the valence electrons as a motif in combination with the highsymmetry lattice. In the case of $\mathrm{Fe}$, it is shown that ferromagnetic ordering reduces the symmetry of the structure from body centered cubic to tetragonal. Thus, the ferromagnetic $\alpha$ - and paramagnetic $\beta$-phase are separate and unique phases that should both be addressed on the $\mathrm{Fe}$ phase diagram. In the case of $\mathrm{Pu}$, first-principles densityfunctional theory calculations are used to show that the anomalously large anisotropy of $\delta$-plutonium is a consequence of greatly varying bond-strengths between the 12 nearest neighbors. Employing the calculated bond strengths in crystallographic determination yields a structure with the monoclinic space group $\mathrm{Cm}$ for $\delta$-Pu rather than face-centered cubic $F m \overline{3} m$. The reduced space group for $\delta$-Pu enlightens why it is the only metal with a monoclinic ground state, why lattice distortions of the metal are viable, and has considerable implications for the behavior of the material as it ages. This paper illustrate how an expansion of classical crystallography that accounts for anisotropic electronic structure can explain complicated materials in a novel way.
\end{abstract}

Keywords:

*Contact Author

Tel: $\quad 925-422-9741$

Fax: $\quad 925-422-6892$

Email: moore78@1lnl.gov 


\section{Introduction}

When determining the space group of a crystal, classical crystallography assumes spherical potentials and thus does not incorporate anisotropic electronic structure within its framework [1]. For some metals, such as aluminum, this is certainly justifiable. However, a number of metals have complicated electronic structures, leading to a myriad of abnormal physical properties. For example, upon heating iron transforms from bodycentered cubic (bcc) to face-centered cubic (fcc), then back to bcc. This reversion back to the ground-state phase is peculiar. In addition, there is a magnetic-nonmagnetic transition in bcc iron prior to the transformation to fcc, caused by a collective ordering of the valence electron spins.

Further down the Periodic Table the lanthanides and actinides possess $f$ electrons at or near the bonding energy level. The $f$-electron wave function is highly anisotropic and displays varying degrees of localization of the states [2]. Accordingly, the behavior of $f$-electron metals is considerably more complex than say, aluminum. In the case of iron and plutonium, a more in-depth treatment of the space-group determination of the crystal structure may be in order. With this in mind, we present this paper to illustrate a novel treatment of crystallographic determination of complex materials with anisotropic valence-electron behavior. For this task we have chosen two complicated metals; one with a magnetic-nonmagnetic transition - iron, and one with highly anisotropic bonding plutonium. Our goal is to impress upon the reader that this treatment offers a fresh way to interrogate complicated metals, yielding a novel way to address outstanding, and in the case of iron old, questions. 


\subsection{Iron}

The paramagnetic $\beta$ phase in Fe was removed many years ago from the phase diagram and replaced with the ferromagnetic $\alpha$-Fe, due to the belief that they did not constitute separate phases of the metal. This has produced considerable confusion among the metallurgical community, spawning numerous heated arguments. As far as could be determined by x-ray diffraction at the time [3], the structure of ferromagnetic Fe was the same as that of paramagnetic Fe. Thus, no change of phase was deemed to have occurred when Fe lost its magnetism at the Curie temperature.

Differences in magnetic behavior arise from differences in magnetocrystalline anisotropy and exchange interactions for different materials. Magnetocrystalline anisotropy is linked to the symmetry of the crystal and involves the electron spin-orbital interactions and crystal fields [4,5]. Magnetocrystalline anisotropy generates the directions for the magnetic moments that have minimum energy. The exchange interactions are quantum mechanical phenomena required by the Pauli exclusion principle. For strong exchange, the magnetic moments have a strong coupling to one another and is cooperative phenomena. Small exchange interactions yield noncooperative phenomena. In the case of strong exchange, the valence electrons behave in a highly anisotropic manner, one which affects the overall symmetry of the structure.

The question still stands whether paramagnetic $\beta$-Fe and ferromagnetic $\alpha$-Fe each constitute a distinct crystal structure, even if the lattice remains bec where $a=b=c$. 


\subsection{Plutonium}

Metals that straddle the magnetic-superconducting transition exhibit a fascinating interplay of characteristics, such as itinerant magnetism, heavy fermions, superconductivity, and phase instability [6-10]. A rearranged period table is shown in Figure 1 containing the five transition metal series; $4 f, 5 f$, and $3 d$ to $5 d$ [11]. At ground state, the metals in the blue area exhibit superconductivity and the metals in the red area exhibit a magnetic moment. The white band is a transition region where metals are on the borderline between localized (magnetic) to itinerant (conductive) valence-electron behavior. On this transition, metals such as cerium, neptunium, plutonium, and iron are found, each of which can be categorized as a complex metal. Of these metals, plutonium is arguably the most complex with a perplexing electronic structure, and, in turn, a myriad of unique properties.

Plutonium exhibits six solid allotropic phases $\alpha, \beta, \gamma, \delta, \delta^{\prime}$, and $\varepsilon$, where the volume of the crystal first expands upon heating then condenses, as shown in Figure 2 $[2,12]$. The $\alpha$ and $\beta$ phase are monoclinic, the $\gamma$ phase is orthorhombic, the $\delta$ phase is fcc, the $\delta^{\prime}$ phase is tetragonal, and finally the $\varepsilon$ phase is bcc. This is followed by the liquid phase, which not only emanates at a very low temperature for a metal $\left(\sim 640^{\circ} \mathrm{C}\right)$, but also is more dense than the previous phase, similar to $\mathrm{H}_{2} \mathrm{O}$. Also to note from Figure 2 is that $\delta$ and $\delta^{\prime}$ have a negative coefficient of thermal expansion, a quite unusual property for any metal. The oddity of $\mathrm{Pu}$ is further illustrated in the pseudo-binary phase diagram of the light actinides in Figure 3. Note how both the melting temperature drops and the 
number of solid allotropic phases increases along the series when approaching $\mathrm{Pu}$. This reveals that $\mathrm{Pu}$ is at the zenith of complex behavior for the $5 f$ metal series.

The phonon dispersion curves for $\delta$-plutonium provide yet another intriguing characteristic of the metal. Inelastic X-ray scattering recorded from single-grains regions of $\delta$-plutonium [13], as well as other measurements $[14,15]$, show that it is the most anisotropic face-centered cubic (fcc) metal known. The shear moduli $\mathrm{C}_{44}$ and $\mathrm{C}^{\prime}$ differ by a factor of $\sim 7$, which is in strong contrast to aluminum exhibiting a factor of 1.2 [16]. This evidence, in conjunction with the highly anisotropic nature of the $f$-electron wave function, implies that the bonding strengths between the 12 nearest neighbors of the fcc $\delta$-Pu lattice are not equal. In turn, this means that the total symmetry of the metal may not be fcc, but rather a lower symmetry class.

\subsection{Goals of this paper}

First: To illustrate that augmenting classical crystallography to account for anisotropic valence electron behavior is feasible and can explain perplexing behavior of complex materials in a novel way. In order to do this, we address two elements that reside on the superconducting-magnetic transition in Figure 1; iron and plutonium.

Second: To show that the paramagnetic $\beta$-phase of iron is rightly a structure unto itself that should be incorporated into the iron phase diagram. The magnetic moment on each atom of ferromagnetic $\alpha$-Fe reduces the total symmetry of the structure, in turn altering the macroscopic physical properties we observe, such as elastic constants, shear modulus, and phase transformations. $\beta$-Fe is bcc; $\alpha$-Fe is tetragonal. 
Third: To systematically progress through crystallographic arguments showing that $\delta$-Pu belongs to the monoclinic space group $C m$ rather than the cubic space group $F m \overline{3} m$. This is achieved using first-principles density-functional theory calculations in a novel way to obtain the bond-strengths between the 12 nearest neighbors. Our results provide new insight into why plutonium is highly anisotropic, why it is the only metal with a monoclinic ground state, and why tetragonal, orthorhombic, or monoclinic distortions of $\delta$ $\mathrm{Pu}$ are likely.

\section{Calculation methods}

For Pu bond strengths, full-potential linear-muffin-tin orbital FPLMTO calculations [17] were employed, since these have been used extensively and successfully for transition [18] and actinide [19] metals. The "full potential" refers to the use of non-spherical contributions to the electron charge density and potential. This is accomplished by expanding these in cubic harmonics inside non-overlapping muffin-tin spheres and in a Fourier series in the interstitial region. We use two energy tails associated with each basis orbital and for the semi-core $6 s, 6 p$, and valence $7 s, 7 p, 6 d$, and $5 f$ states, these pairs are different. Spherical harmonic expansions are carried out through $1_{\max }=6$ for the bases, potential, and charge density. For the electron exchange and correlation energy functional, the generalized gradient approximation (GGA) is adopted [20,21]. The nearest-neighbor bond strengths are obtained from total energy calculations of a 27-atom super cell that uniquely defines all 12 nearest neighbors (NN). By introducing a small (2\%) displacements, along each of the $12 \mathrm{NN}$ bonds, the force associated with the respective bond is obtained from the corresponding energy shift scaled by the magnitude 
of the displacement. This is the 'bond strength.' The super-cell is allowed to spin polarize ferromagnetically, whereas spin-orbit interaction is omitted to simplify the calculations. It has been shown that spin-orbit interactions are strong in the $5 f$ states of the actinides $[22,23]$. However, the spin-orbit splitting that occurs in the $5 f$ states can be mimicked by ferromagnetic ordering [24]. While not strictly correct, since experiments show $\delta$-Pu is nonmagnetic, the ferromagnetic ordering greatly simplifies the complex and time-consuming calculations. Integration over the irreducible wedge of the Brillouin zone (IBZ, $1 / 4^{\text {th }}$ of the full zone) is performed using the special k-point method [25] and we used $20 \mathrm{k}$ points in the IBZ for the fcc super-cell.

\section{Results}

In order to progress our argument that classical crystallography can be augmented to account for anisotropic valence electron behavior, we will begin with a two-dimensional plane group then proceed to three-dimensional space groups. Both $\mathrm{Fe}$ and $\mathrm{Pu}$ will be considered in the progression of this argument, but each will be addressed separately in the discussion section.

Let us begin with a two-dimensional array of blocks, as shown in Figure 4 (a and b). First, a square array of points is created, which is the lattice. Blocks are then incorporated around each lattice point, which is the motif. The resulting structure is shown Figure 4 (a). The combination of a lattice and motif in two dimensions results in a plane group, the two-dimensional analog to a space group for three-dimensions. This two-dimensional structure in Figure 4 (a) has a plane group symmetry of $4 \mathrm{~mm}$ because there is an axis of 4-fold rotation about each lattice point and there are four mirror planes 
marked $m_{1}, m_{2}, m_{3}$, and $m_{4}$. When the upper-left and lower-right corners of each block are filled, the motif is altered. The plane group symmetry of this structure is accordingly reduced to $2 \mathrm{~mm}$ because of the loss of 4-fold symmetry and the elimination of the $m_{1}$ and $m_{2}$ mirror plane planes. The important point is that the structure in 4 (b) still has a square lattice, even though the axis of 4-fold symmetry is lost and the total symmetry of the structure is reduced.

Body-centered cubic Fe can be utilized as a three-dimensional real crystal structure to progress this idea. When paramagnetic, the structure has a bcc space group of $\operatorname{Im} \overline{3} \mathrm{~m}$. However, when the crystal structure becomes ferromagnetic with aligned moments in the [001] direction the symmetry is reduced to $I 4 / \mathrm{mm}^{\prime} \mathrm{m}^{\prime}$, as shown in Figure 5 [13]. From a geometry standpoint $a=b=c$, but the fact that the spins are aligned along the $z$-axis lowers the symmetry of the crystal structure. Thus, there does not need to be atomic displacements, and correspondingly a change in lattice, to reduce the symmetry of the structure.

In the case of the two-dimensional array of blocks in Figure 4 (plane group) or in the three-dimensional bcc structure of Fe in Figure 5 (space group), the same point comes out: the lattice itself may remain high symmetry, but the crystal structure as a whole may be reduced in symmetry due to a change in motif. This is because the symmetry of any plane or space group is composed of the intersection of the symmetries of a lattice and a motif. In ferromagnetic $\alpha$-iron, the magnetic moment produced by the outermost valence electrons not only degrades the symmetry from bcc to tetragonal, but also alters the macroscopic properties that we observe, such as elastic constants, shear modulus, and phase transformations. While a magnetic moment can reduce symmetry as described above, other aspects of the outermost bonding electrons can also affect the symmetry. 
Armed with this knowledge, let us return to the case of $\delta$-Pu. We begin with the assumption that the lattice is fcc with $a=b=c$. A motif is then created, which is the bond strengths for the 12 nearest neighbors. The question is, if an fcc lattice is joined with a motif of anisotropic bonds, what is the resultant space group? In order to resolve this issue the detailed electronic structure of $\mathrm{Pu}$ must be determined, and in particular the variation of the nearest-neighbor bond strengths. This was performed by calculating the energy response $(\Delta \mathrm{E})$ of a $2 \%$ displacement of an atom along each of the 12 nearestneighbor directions and scaling this with the displacement magnitude $(\mathrm{u}=0.049 \AA)$, as described in the methods section. Density-functional theory has proven to be accurate for most metals in the Periodic Table, including $\mathrm{Pu}$ [26], however, its use for calculating bond strength is entirely unique.

The results of the calculations are shown in Table 1 and Figure 6. The nearest neighbor direction $(x, y, z)$, Miller indices $(h, k, l)$, change in energy $(\Delta \mathrm{E})$ due to a $2 \%$ shift of the $0,0,0$ atom, and the change in energy normalized by the displacement length $(\Delta \mathrm{E} / \mathrm{u})$ are shown in Table 1. Notice how $\Delta \mathrm{E} / \mathrm{u}$ varies from $\sim 3.3$ to $\sim 5.3$, showing the large degree of variation in bond strength between the 12 nearest neighbors. As shown in Figure 6, the twelve nearest neighbors can be separated into six pairs of two where the bond strengths are close in value: blue (3.3), black (3.5-3.7), red (3.7-3.9), pink (3.9-4.1), green (4.5-4.7), and brown (4.7-5.3). In the (001) plane, the [110] bond is roughly equal to the [110] bond (green), and the [ $\overline{110}$ bond is roughly equal to the [110] bond (black).

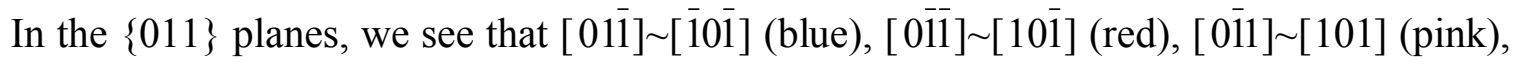
and $[011] \sim[101]$ (brown). It is important to note that not only the bond strength, but also the repeatability of groupings dictate the choice of sets. In other words, there is a clear separation between the brown and pink sets and the clear separation between the red and 
blue sets. It is interesting that the bonds in the (001) plane are almost equal directly across the central atom, whereas the bonds in the $\{011\}$ planes are not and have a more complicated arrangement.

When an fcc lattice is joined with the calculated bond strengths as a motif, the resultant structure is $c$-centered monoclinic with the space group $\mathrm{Cm}$. This low symmetry space group is due to the fact that besides translational symmetry there is no rotational symmetry and only one mirror plane along the (110) plane. The new Bravais lattice for the structure is shown in Figure 7, where (a) is the three-dimensional rendering of the $c$ centered unit cell and (b) is a two-dimensional rendering viewed along the [001] direction. In (b) the fcc lattice is shown by the purple box. The $c$-centered unit cell is shown by the heavy red box with the green line marking the [110] mirror plane.

This space group has several ramifications. First, it now seems no coincidence that the ground state of $\mathrm{Pu}$ is monoclinic $C 2 / m$ and that here we show $\delta$-Pu exhibits a monoclinic space group of $\mathrm{Cm}$ when the bonding strengths are accounted for as the motif. The only difference between the two space groups $C 2 / m$ and $C m$ is an axis of two-fold rotation perpendicular to the mirror plane. (Of course, in the real structures there is also a slight change of atomic positions). A reduced space group of $\mathrm{Cm}$ for $\delta$-Pu also yields a viable path for the $\delta \leftrightarrow \alpha^{\prime}$ phase transformation of $\mathrm{Pu}$ and $\mathrm{Pu}$ alloys.

A second important consequence of these calculations is that the structure is not centrosymmetric (defined as: $x=-x ; y=-y ; z=-z$ ). Classical crystallography assumes spherical atoms occupy each lattice site and that fcc crystals are centrosymmetric However, the above arguments show that this is a flawed idea for $\delta$-Pu. The electronic structure (dominated by $5 f$ states) produces bonding in $\delta$-Pu with largely varying strength 
between the 12 nearest neighbors, thus making the crystal highly anisotropic, and one that is the least applicable candidates for centrosymmetry compared to other fcc metals.

\section{Discussion}

The crystallographic arguments set forth in the Results section illustrate how anisotropic behavior of valence electrons can degrade the overall symmetry of a given crystal structure. Again, this is due to the fact that the symmetry of a real crystal is given by the intersection of the lattice and motif. In reality, each atom is the motif, hung on a given lattice. Ferromagnetic Fe is an atom with aligned magnetic moment hung on a bcc lattice. $\delta$-Pu is an atom with highly anisotropic bonding hung on an fcc lattice. Taking into account these behaviors - ferromagnetism and anisotropic bonding - we find that the total symmetry of the structure is reduced. The reduced symmetry can be used to explain perplexing aspects of complex metals. This idea is novel and surely creates a plethora of questions. In the flowing discussion we will address the questions set forth in the introduction and ones which naturally evolved in progress of this work.

\subsection{Iron}

In the introduction the question was raised; 'is paramagnetic $\beta$-Fe a separate phase from ferromagnetic $\alpha$-Fe?' The answer is yes. The electron spins align in $\alpha$-Fe along the $z$-axis creating a moment that changes the motif of the structure. Irregardless whether the bcc lattice retains the high symmetry parameters $a=b=c$, the total symmetry 
is indeed lowered from $\operatorname{Im} \overline{3} \mathrm{~m}$ to $I 4 / \mathrm{mm}^{\prime} \mathrm{m}^{\prime}$ due to the aligned electron spins.

It has been shown that magnetic point groups can be used to describe the symmetry of crystals that have magnetic moments associated with the atoms [27]. In this treatment, phenomena in magnetic materials, such as magnetic domains, domain interactions, magnetocrystalline anisotropy, and magnetostriction are shown to be more accurately addressed by using the full symmetry aspects of the magnetic state, i.e., the structure with reduced symmetry due to the magnetic ordering. Electronic structure calculations are also better performed when the symmetry is reduced due to the ordered magnetic state. Accurate orbital magnetic moments of bcc iron can only be obtained from calculations [28] that consider a lowering of the symmetry, from 48 (cubic) to 16 (tetragonal) operations, due to the preferred orientation of the magnetic spin moments along the [001] easy axis. Cubic symmetry does not support ferromagnetism because the moments always produce a special direction, as shown in Figure 5.

Although not cubic, PrCos has yielded experimental evidence of symmetry reduction upon magnetic ordering. Shen and Laughlin [29] have shown by convergent beam electron diffraction that the projected point group symmetry along the [0001] direction of $\mathrm{PrCo} 5$ is reduced from $6 \mathrm{~mm}$ to 6 on magnetic ordering. This implies that the space group changed from $P 6 / \mathrm{mmm}$ in the paramagnetic state to $P 6 / \mathrm{mm}$ 'm' in the ferromagnetic state.

\subsection{Plutonium}


The calculations and crystallographic arguments above show that $\delta$-Pu is $c$ centered monoclinic with the space group $\mathrm{Cm}$ when the anisotropic bonds are taken into account. This idea is supported by several of the macroscopic physical properties of the metal. First, $\delta-\mathrm{Pu}$ is most crystallographically expanded phase of all six allotropes. This is opposite of almost all other metals in the Periodic Table, where the fcc phase is the most compact and densely packed. This is support that the Pu atom can not be safely assumed to be a hard sphere as often done. Second, the coefficient of thermal expansion for $\delta$ - $\mathrm{Pu}$ is negative. This is likely caused by anisotropic distribution of the valence electron charge density, again making a hard sphere assumption inappropriate. Together, the low density and negative thermal expansion coefficient can be taken as physical affects due to the reduction of symmetry of $\delta$-Pu.

The reduction of symmetry of $\delta$-Pu illustrated above clarifies recent experimental results, such as those from Lawson et al. [30,31] that suggest a tetragonal distortion occurs in Ga-stabilized $\delta$-Pu. In the work by Lawson et al. [30,31], neutron diffraction revealed that the widths of the peaks were temperature dependent for $\mathrm{Pu}_{0: 98} \mathrm{Ga}_{0: 02}$. As the sample was cooled, peak broadening occurred, increasing with subsequent cooling cycles. This affect disappeared when heated to $650 \mathrm{~K}$. The diffraction peaks were anisotropic, which were modeled by assuming a small tetragonal distortion to the crystal. Because the changes of the diffraction peaks were observed at low temperature, it is possible that they are due to the anisotropic response of the $\delta$-Pu matrix to the ingrowth of monoclinic $\alpha^{\prime}-\mathrm{Pu}$ particles that form during an isothermal martensitic phase transformation at low temperatures [32]. If one introduces precipitates or defects such as vacancies, interstitials, dislocations, and/or He bubbles [33,34] into an anisotropic medium, the variation in bond strength will cause the lattice to extend more in the soft 
directions and less in the rigid directions. Thus, tetragonal, orthorhombic, or monoclinic distortions in aged Ga-stabilized $\delta$-Pu are logical, because precipitation of second-phase particles or the accumulation of damage will strain the lattice and contract/expand it disproportionately in different directions. Also to note is the small value of $\mathrm{C}^{\prime}$ for $\delta$ $\mathrm{Pu}[13,35,36]$, which implies a soft response of the system to a volume-conserving tetragonal distortion.

Another interesting observation was that of Cox et al. [37], where extended X-ray absorption fine-structure spectroscopy was applied to Ga-stabilized $\delta$-Pu. In these experiments it was observed that the local structure of plutonium about the Ga atoms was well defined and quite similar to a typical fcc metal. However, the local structure of plutonium around $\mathrm{Pu}$ atoms was disordered, especially in the individual shells at radii greater than $3.5 \AA$. This means that there is a complexity in the distribution of the neighboring atoms in the successive shells around each $\mathrm{Pu}$ atom, and the authors ascribe this to local distortion of the Pu-Pu bonds in the vicinity of $\mathrm{Ga}$ impurities. This again shows that when under stress, $\delta$-Pu distorts in such a way as to lose its fcc crystal structure, likely due to the 12 nearest neighbor bonds having markedly different strengths.

The two examples above were for $\delta$-Pu in a perturbed system, and its anisotropic response. However, the question arises what does the symmetry of $\delta$-Pu appear as experimentally while under normal conditions? This can be interrogated using highresolution synchrotron-radiation-based x-ray diffraction. While x-ray diffraction does not measure bond energies, it does measure electron charge density. The x-ray scattered intensity for a given reflection is determined by the Fourier transform of the charge density [38]. In the case where there is a spherical (isotropic) and non-spherical 
(anisotropic) part, each can be separated and calculated. The spherical part will be much larger than the non-spherical part, and a rough calculation of the difference in intensity can be obtained by $(1 / Z)^{2}$. In the case of $\mathrm{Pu}$, any additional reflection due to anisotropic distribution of the valence electrons would be approximately $1 \times 10^{-4}$ weaker than the primary reflections. In reality, additional reflections are probably more on the order of 1 $\times 10^{-5}$ to $1 \times 10^{-6}$ weaker, and the above simple equation is more accurate for light elements. We predict that either 1) additional reflections or 2) highly asymmetric primary reflections should occur due to a reduction of symmetry, and that these effects should be detectable with present synchrotron sources and detectors. We have attempted detection of extra reflections using electron diffraction in a transmission electron microscope (TEM). However, high thermal diffuse scattering, large amounts of double diffraction due to the high atomic number, and omnipresent surface oxidation have precluded the ability to do this. For these reasons, x-ray diffraction of large, single-grain samples performed at low temperatures will be the appropriate experiment.

4.3. Given anisotropic valence electron distribution, can a high-symmetry lattice be retained?

When an aligned magnetic moment or highly anisotropic bonds are present in a structure can a high-symmetry lattice be retained or do these affects naturally distort the lattice to a lower symmetry? The answer to this question is not clear. In the case of Fe it has been reported that a single crystal and single magnetic domain of $\mathrm{Fe}$ has $\mathrm{c} / \mathrm{a}=1.3 \mathrm{x}$ $10^{-5}$ [39]. If correct, this shows the lattice has degraded from bcc to tetragonal. However, is this reduction of symmetry a consequence of the aligned magnetic moments, 
which strain the lattice and distorts it to tetragonal?

In the case of $\mathrm{Pu}$ the synchrotron radiation-based x-ray diffraction experiment above was suggested in order to find additional or asymmetric primary reflections due to anisotropic valence electron distribution. However, it is also possible that the lattice is not fcc and there is a very small distortion to a lower symmetry lattice due to the varying bond strength between the 12 nearest neighbors. This again will need to be addressed with high resolution $\mathrm{x}$-ray diffraction experiments.

The crystallographic determination of $\alpha-\mathrm{Fe}$ and $\delta$-Pu in the Results section showed there is a symmetry reduction of the total structure solely due to an anisotropic motif, i.e., the lattice remained high symmetry. However, a highly anisotropic motif in the form of a strong magnetic moment or varying bond strengths may naturally cause a reduction of the lattice symmetry unless some other forces within the crystal can compensate for the anisotropic valence electron influences. This matter surely requires more experiments and calculations aimed at answering these questions.

\subsection{Do fcc structures really exist?}

Given the above results and discussion one should naturally ask 'do fcc crystal structures really exist?' The answer is no. There are certainly fcc lattices, since a lattice is a theoretical construct of points in space. When atoms are centered on these points, then a crystal can indeed exhibit an fcc lattice. However, when a lattice and a motif are intersected, and the full symmetry of each atom is accounted for on that lattice site, there is no true fcc crystal structure. This is because no atom, even Al, has a truly spherical 
potential as assumed in classical crystallography, but rather some anisotropic directionality in the distribution of electron charge density.

If one is to imagine a spectrum of elastic isotropy, Al would reside on one side and plutonium on the other. With this in mind, we performed the same calculations on $\mathrm{Al}$ as done for $\mathrm{Pu}$, which is well known to be the most isotropic fcc metal [40,41]. The results, which are in the same units as the Pu results, lie within 7.55-7.57 for the nearestneighbor bond strengths. The slight variation in the resulting numbers is numerical noise. This shows that any directionality in bond strength is below the detectibility of the calculations, and shows the highly isotropic nature of Al. Nonetheless, we do know that there is a small amount of anisotropy, since the shear moduli $\mathrm{C}_{44}$ and $\mathrm{C}^{\prime}$ in aluminum differ by 1.2 [16]. Thus, even $\mathrm{Al}$ on the far end of the spectrum of elastic isotropy shows some anisotropy.

Another way to think of this is idea is to compare it to the construct of bonding, where we categorize atomic bonds as ionic, covalent, or metallic. While there are materials that have bond types that tend strongly towards one type of bonding, there are no pure ionic, covalent, or metallic bonds in nature. Crystals always exhibit some mixture of the three bond types, since each bond types represents a theoretical end member in a triangular bonding space. In a similar manner, the fcc crystal structure is a theoretical construct with which to aid in the description of real crystal structures. The lattice of a given crystal may be fcc, but the total symmetry of the real structure is not. Looking at the Periodic Table, Al would be the closest to a true fcc structure and $\mathrm{Pu}$ would be the most removed.

While this may seem a rather esoteric discussion, it reveals the need to address the electronic structure of some metals with complex electronic structures, such as Fe, Mn, 
$\mathrm{Ce}, \mathrm{U}$, and $\mathrm{Pu}$, when performing crystallography determination. The calculations and crystallographic determination above for $\mathrm{Pu}$ show that it is the most removed from a true fcc metal and in order to be most accurately presented should be considered monoclinic with the space group $\mathrm{Cm}$. The fact that many of the perplexing physical properties of the metal, such as the monoclinic ground state, are enlightened by this treatment, shows the approach is valid and a novel tool for understanding materials.

\section{Conclusions}

The following conclusions can be drawn from this paper:

1) When anisotropic electronic structure is incorporated in crystallographic determination of a material the total symmetry of the structure is often reduced, even though the lattice may remain high symmetry. This approach can be used to explain perplexing behavior of complex materials in a new way.

2) The $\beta$-phase of iron is a separate structure from ferromagnetic $\alpha$-Fe and should be incorporated in the iron phase diagram. The magnetic moment on each atom in $\alpha$-Fe reduces the total symmetry of the structure from bcc, in turn altering the macroscopic physical properties we observe, such as elastic constants, shear modulus, and phase transformations.

3) First-principles density-functional theory calculations are used in a new way to show that the anomalously large anisotropy of $\delta$-plutonium is a consequence of greatly varying bond-strengths between the 12 nearest neighbors. A systematically progression through crystallographic arguments 
armed with these bond strength shows that $\delta$-Pu belongs to the monoclinic space group $\mathrm{Cm}$ rather than the cubic space group $\mathrm{Fm} \overline{3} m$. The reducedsymmetry structure provides new insight into why $\mathrm{Pu}$ is the only metal with a monoclinic ground state and why tetragonal, orthorhombic, or monoclinic distortions of $\delta$-Pu are likely.

\section{Acknowledgements}

L. Yang, A. Landa, P. Heaney, J. Bradley, D. Farber, M. Wall, and T. Massalski are gratefully acknowledged for helpful discussions. This work was performed under the auspices of U.S. Department of Energy by the University of California, Lawrence Livermore National Laboratory under contract No. W-7405-Eng-48. 


\section{References:}

[1] Bloss FD. Crystallography and Crystal Chemistry. Holt, Rinehart, and Winston Inc. 1971.

[2] Boring AM, Smith JL. Plutonium condensed-matter physics. In Challenges in plutonium science, Volume I, Los Alamos Science, Number 26, 2002.

[3] Westgren A, Phragmén G. J. Iron Steel Inst 1922; 1:241-73.

[4] Hurd CM. Contemp Phys 1982; 23:469.

[5] Coey JMD. J Appl Phys 1978; 49:1646.

[6] Cox L. Phys Rev Lett 1987; 59:1240.

[7] Sarrao JL, Morales LA, Thompson, JD, Scott BL, Stewart GR, Wastin F, Rebizant J, Boulet P, Colineau E, Lander GH. Nature 2002; 420:297.

[8] Brooks MSS, Johansson B, Skriver HL. In Handbook on the Physics and Chemistry of the Actinides, Volume 1, Freeman AJ and. Lander GH editors. Amsterdam: NorthHolland, 1984. p. 153.

[9] Hewson AC. The Kondo Problem to Heavy Fermions. Cambridge University Press, 1993.

[10] Moore KT, Wall MA, Schwartz AJ, Chung BW, Morton SA, Tobin JG, Lazar S, Tichelaar FD, Zandbergen HW, Söderlind P, van der Laan G. Phil Mag 2004; 84:1039.

[11] Smith JL, Kmetko EA. J Less Comm Met 1983; 90:83.

[12] Hecker SS. Metall Mat Trans A 2004; 35:2207. 
[13] Wong J, Krisch M, Farber DL, Occelli F, Schwartz AJ, Chiang TC, Wall M, Boro C, Xu R. Science 2003; 301:1080.

[14] Ledbetter HM, Moment RL. Acta Metall 1976; 24:891.

[15] McQueeney RJ, Lawson AC, Migliori A, Kelley TM, Fultz B, Ramos M, Martinez B, Lashley JC, Vogel SC. Phys Rev Lett 2004; 92:146401.

[16] Stedman R, Nilsson G. Phys Rev 1966; 145:492.

[17] Wills JM, Eriksson O, Alouani M, Price DL. In Electronic Structure and Physical Properties of Solids. Dreysse H, editor. Springer-Verlag, Berlin, 1998.

[18] Ahuja R, Soderlind P, Trygg J, Melsen J, Wills JM, Johansson B, Eriksson O. Phys Rev B 1994; 50:14690.

[19] Soderlind P. Adv Phys 1998; 47:959.

[20] Perdew JP, Chevary JA, Vosko SH, Jackson KA, Pederson MR, Singh DJ. Phys Rev B 1992; 46:6671.

[21] Perdew JP, Burke K, Ernzerhof M. Phys Rev Lett 2001; 87:156401.

[22] Moore KT, Wall MA, Schwartz AJ, Chung BW, Shuh DK, R. K. Schulze, Tobin JG. Phys Rev Lett 2003; 90:196404.

[23] van der Laan G, Moore KT, Tobin JG, Chung BW, Wall MA, Schwartz AJ. Phys Rev Lett 2004; 93:097401.

[24] Tobin JG, Moore KT, Chung BW, Wall MA, Schwartz AJ, van der Laan G, Kutepov AL. Phys. Rev. B 2005; 72:085109.

[25] Chadi DJ, Cohen ML. Phys Rev B 1973; 8:5747.

[26] Söderlind P, Sadigh B. Phys Rev Lett 2004; 92:185702. 
[27] Laughlin DE, Willard MA, McHenry ME. In Phase Transformations and Evolution in Materials. Turchi P, Gonis A, editors. The Minerals, Metals and Materials Society 2000 p. 121.

[28] P. Söderlind, Eriksson O, Johansson B, Albers RC, Boring AM. Phys Rev B 1992; 45:12911.

[29] Shen Y, Laughlin DE. Phil Mag Lett 1990; 62:187.

[30] Lawson AC, Martinez B, Von Dreele RB, Roberts JA, Sheldon RI, Brun TO, Richardson Jr. JW. Phil Mag B 2000; 80:1869.

[31] Lawson AC, Roberts JA, Martinez B, Von Dreele RB, Storey B, Heather ??, Hawkins T, Ramos M, Hampel FG, Davis CC, Pereyra RA, Mitchell JN, Freibert F, Valone SM, Claytor TN, Viskoe DA, Schonfeld FW. Phil Mag B 2007; 85:2007.

[32] Zocco TG, Stevens MF, Adler PH, Sheldon RI, Olson GB. Acta Metall Mater 1990; $38: 2275$.

[33] In refs. 30 and $31,{ }^{242} \mathrm{Pu}$ was used, which does not strongly self-irradiate. Thus, the lattice distortions are likely due to the isothermal martensitic phase transformation of $\alpha^{\prime}-\mathrm{Pu}$ particles at low temperatures.

[34] Schwartz AJ, Wall MA, Zocco TG, Wolfer WG. Phil Mag 2005; 85:479.

[35] Dai X, Savrasov SY, Kotliar G, Migliori A, Ledbetter H, Abrahams E. Science 2003; 300:953.

[36] Robert G, Pasturel A, Siberchicot B. J Phys Con Mat 2003; 15:8377.

[37] Cox LE, Martinez R, Nickel JH, Conradson SD, Allen PG. Phys Rev B 1995; 51:751.

[38] Coppens P. X-ray charge densities and chemical bonding. Oxford University Press, 1997. 
[39] Shaskolskaya S. Fundamentals of Crystal Physics. Chapter VIII. Mir Publishers, Moscow, 1982.

[40] King HW, J Mater Sci 1966; 1:79.

[41] Predel B, Gust W. Mater Sci Eng 1972; 10:211. 
Table 1. The nearest neighbor direction in $x, y, z$ coordinates, Miller indices $(h, k, l)$, change in energy $(\Delta \mathrm{E})$ due to a $2 \%$ shift of a $0,0,0$ atom, and the change in energy normalized by the displacement length $(\Delta \mathrm{E} / \mathrm{u})$.

\begin{tabular}{|c|c|c|c|}
\hline $\begin{array}{c}\text { Nearest Neighbor } \\
(x, y, z)\end{array}$ & $\begin{array}{c}\text { Miller Indices } \\
(h, k, l)\end{array}$ & $\begin{array}{c}\Delta \mathrm{E} \\
(\mathrm{mRy} / \text { atom })\end{array}$ & $\Delta \mathrm{E} / \mathrm{u}$ \\
\hline $\begin{array}{lll}0.5 & 0.5 & 0\end{array}$ & 110 & 0.230 & 4.69 \\
\hline$-0.5-0.50$ & $-1-10$ & 0.220 & 4.49 \\
\hline $0.5-0.50$ & $1-10$ & 0.180 & 3.67 \\
\hline $\begin{array}{lll}-0.5 & 0.5 & 0\end{array}$ & -110 & 0.170 & 3.47 \\
\hline $\begin{array}{lll}0.5 & 0 & 0.5\end{array}$ & 101 & 0.200 & 4.08 \\
\hline $\begin{array}{lll}-0.5 & 0 & -0.5\end{array}$ & $-10-1$ & 0.160 & 3.27 \\
\hline$-0.5 \quad 0 \quad 0.5$ & -101 & 0.230 & 4.69 \\
\hline $\begin{array}{lll}0.5 & 0 & -0.5\end{array}$ & $10-1$ & 0.190 & 3.88 \\
\hline $\begin{array}{lll}0 & -0.5 & 0.5\end{array}$ & $0-11$ & 0.190 & 3.88 \\
\hline $0 \quad 0.5-0.5$ & $01-1$ & 0.160 & 3.27 \\
\hline $\begin{array}{lll}0 & 0.5 & 0.5\end{array}$ & 011 & 0.260 & 5.31 \\
\hline $0 \quad-0.5-0.5$ & $0-1-1$ & 0.180 & 3.67 \\
\hline
\end{tabular}




\section{Figure captions}

Figure 1. A rearranged periodic table where the five transition metal series $4 f, 5 f$, and $3 d$ to $5 d$ are shown (after [11]). When cooled to the ground state, the metals in the blue area exhibit superconductivity while the metals in the red area exhibit magnetic moments. The white band running though the middle is where conduction electrons transition from itinerant and pairing to localized and magnetic. Slight changes in temperature, pressure, or chemistry will move metals located on the white band to either more bonding or more magnetic behavior. The two metals examined here, $\mathrm{Fe}$ and $\mathrm{Pu}$, are cirled in green and show there relation to the magnetic-superconducting transition.

Figure 2. The Pu phase diagram showing the large number of solid allotropic phases, the negative coefficient of thermal expansion exhibited by $\delta$ and $\delta^{\prime}$, and the density increase that occurs upon melting.

Figure 3. A pseudo-binary phase diagram of the light actinide metals near Pu (after [2]). Notice the increase in the number of solid allotropic phases and the decrease in melting temperature that occur near $\mathrm{Pu}$.

Figure 4. Two periodic arrays illustrating repetition of a lattice (points) and a motif (blocks). (a) a pattern showing a plane group symmetry of $4 \mathrm{~mm}$ and (b) a pattern showing a plane group symmetry of $2 \mathrm{~mm}$, reduced only by a change in the motif, not the lattice. 
Figure 5. The structure of $\alpha$-iron. The crystal has a body-centered cubic space group of $\operatorname{Im} \overline{3} m$ when paramagnetic, but is reduced to $I 4 / \mathrm{mm}^{\prime} m^{\prime}$ when the crystal becomes ferromagnetic with aligned moments in the [001] direction. Note that the atomic positions do not change. Rather, the motif is changed by the addition of an ordered magnetic moment as indicated in the upper-right inset of the figure.

Figure 6. Two stacked fcc unit cells with the central atom showing the 12 nearest neighbors. In the case of plutonium, the 12 bonds with the nearest neighbors widely vary with strength and can be separated into six pairs: blue (3.3), black (3.5-3.7), red (3.7-3.9), pink (3.9-4.1), green (4.5-4.7), and brown (4.7-5.3). When the fcc lattice is combined with the motif of these bond strengths the resultant space group is monoclinic $\mathrm{Cm}$.

Figure 7. (a) Three-dimensional and (b) two-dimensional representations of the ccentered monoclinic Bravais Lattice needed to fully describe $\delta$-Pu. 


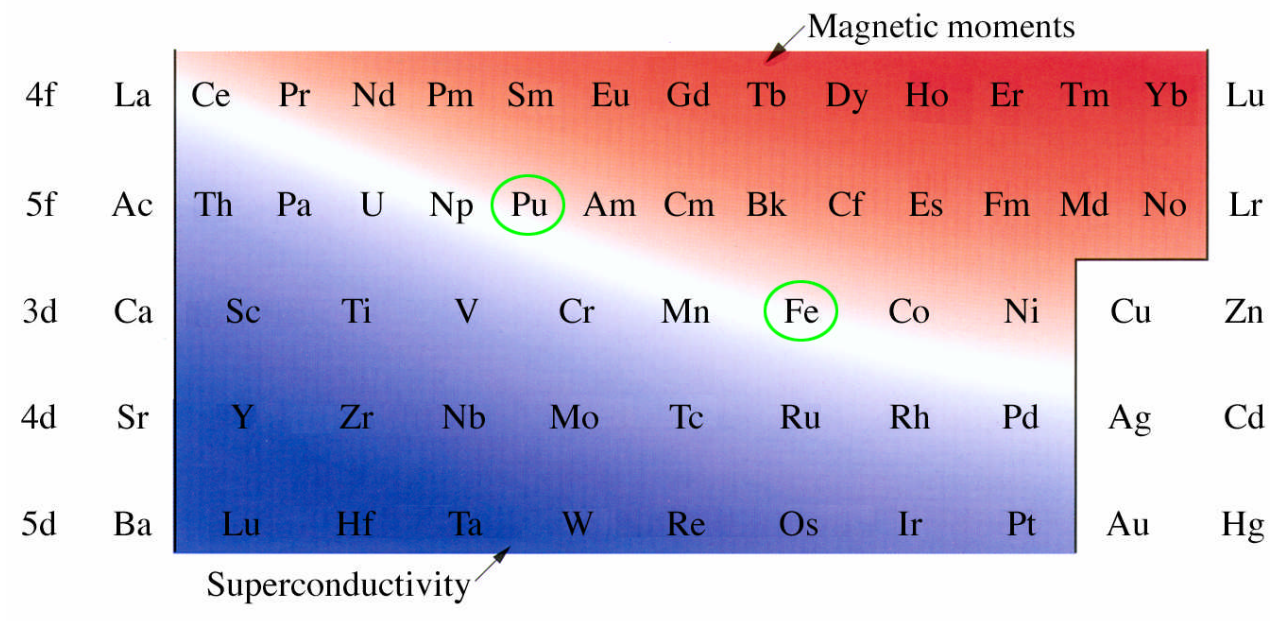

Figure 1

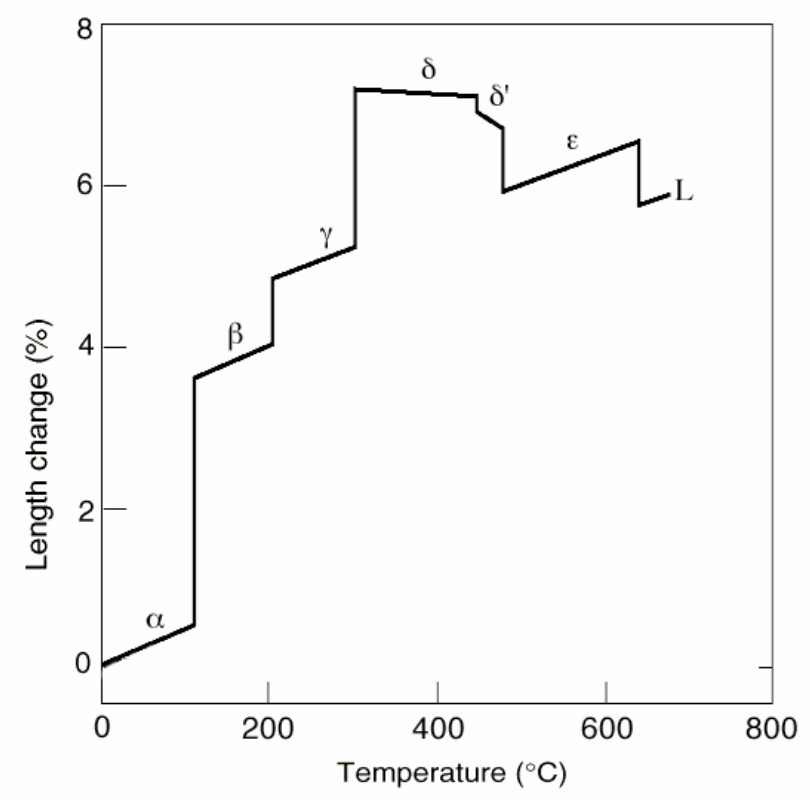

Figure 2 


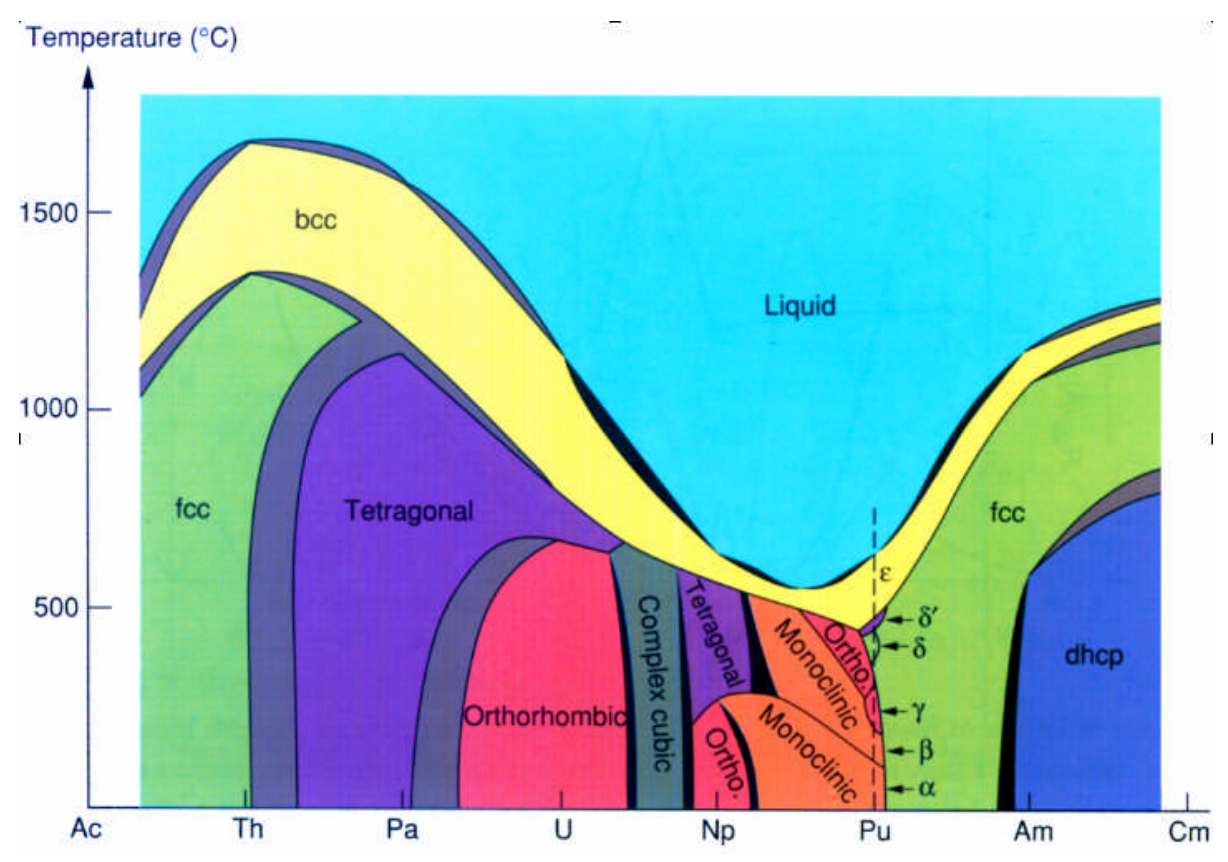

Figure 3
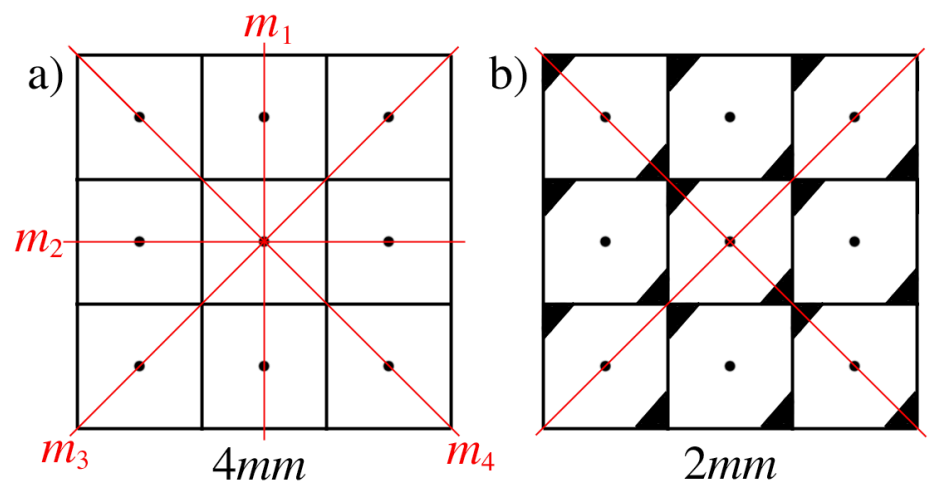

Figure 4 


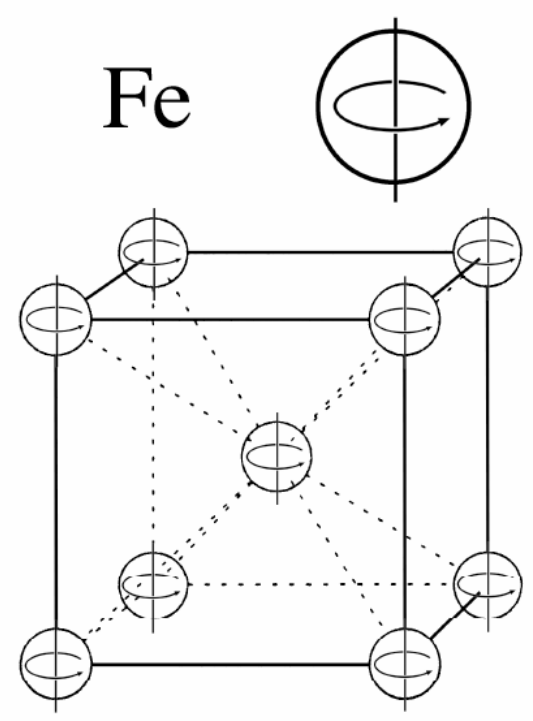

Figure 5

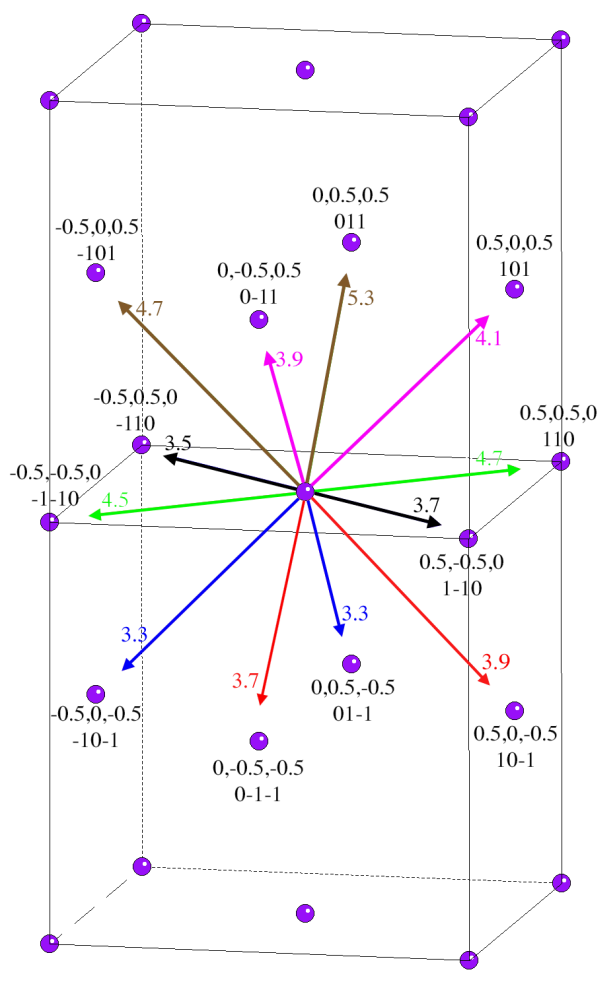

Figure 6 
a)

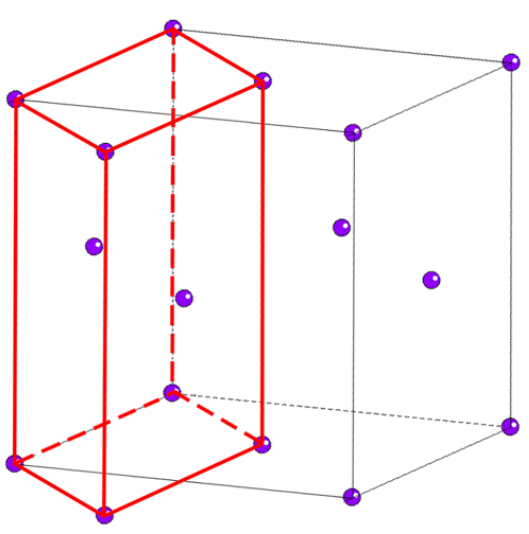

b)

$$
\begin{aligned}
& \text { - } 002 \text { plane } \\
& \text { - } 001 \text { plane }
\end{aligned}
$$
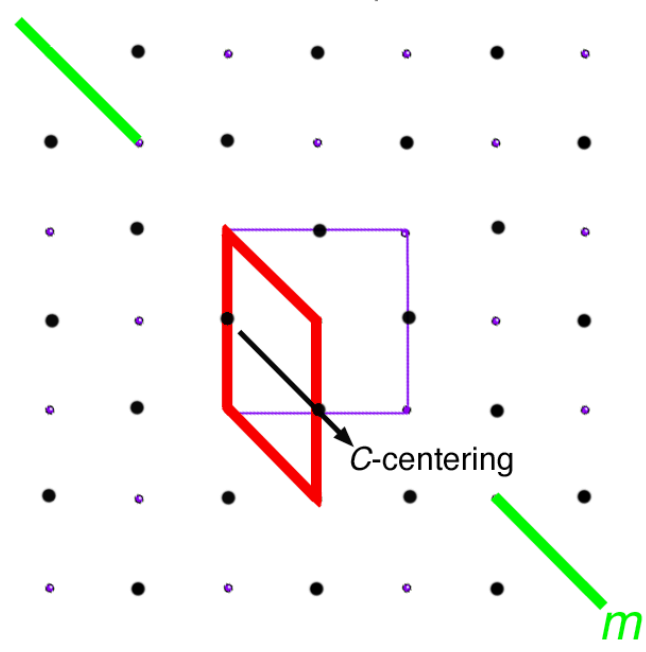

Figure 7 